\title{
Co-morbidity of personality disorder in schizophrenia among psychiatric outpatients in China: data from epidemiologic survey in a clinical population
}

\author{
YanYan Wei ${ }^{1,2+}$, TianHong Zhang ${ }^{2 \dagger}$, Annabelle Chow ${ }^{3}$, YingYing Tang ${ }^{2}$, LiHua Xu ${ }^{2}$, YunFei Dai ${ }^{2}$ XiaoHua Liu ${ }^{2}$, \\ Tong Su${ }^{1}$, Xiao Pan ${ }^{1}$, Yi Cui ${ }^{1}$, ZiQiang Li ${ }^{1}$, KaiDa Jiang ${ }^{2}$, ZePing Xiao ${ }^{2}$, YunXiang Tang ${ }^{{ }^{*}}$ and JiJun Wang ${ }^{2,4^{*}}$
}

\begin{abstract}
Background: The reported rates of personality disorder (PD) in subjects with schizophrenia (SZ) are quite varied across different countries, and less is known about the heterogeneity of PD among subjects with SZ. We examined the co-morbidity of PD among patients who are in the stable phase of SZ.

Method: 850 subjects were randomly sampled from patients diagnosed with SZ in psychiatric and psycho-counseling clinics at Shanghai Mental Health Center. Co-morbidity of PDs was assessed through preliminary screening and patients were administered several modules of the SCID-II. Evidence of heterogeneity was evaluated by comparing patients diagnosed with SZ with those who presented with either affective disorder or neurosis (ADN).

Results: 204 outpatients (24.0 \%) in the stable phase of SZ met criteria for at least one type of DSM-IV PD. There was a higher prevalence of Cluster-A (odd and eccentric PD) and C (anxious and panic PD) PDs in SZ (around $12.0 \%$ ). The most prevalent PD was the paranoid subtype (7.65\%). Subjects with SZ were significantly more likely to have schizotypal PD ( $4.4 \%$ vs. $2.1 \%, p=0.003$ ) and paranoid PD (7.6 \% vs. $5.4 \%, p=0.034)$, but much less likely to have borderline, obsessive-compulsive, depressive, narcissistic and histrionic PD.

Conclusions: These findings suggest that DSM-IV PD is common in patients with SZ than in the general population. Patterns of co-morbidity with PDs in SZ are different from ADN.
\end{abstract}

Keywords: Schizotypal personality disorder, Prevalence, Cluster-A personality disorders, Psychosis, Clinical population

\section{Background}

There is a close relationship between personality disorder (PD) and schizophrenia (SZ). This relationship is supported by epidemiological, phenomenologic, and biologic studies [1-6]. However, patients with SZ do not appear to adhere to the normative pattern of co-morbidity. Research has

\footnotetext{
*Correspondence: tangyun7633@sina.com; jijunwang27@163.com

${ }^{\dagger}$ Equal contributors

'Department of Medical Psychology, Faculty of Mental Health, Second Military Medical University, Shanghai 200433, People's Republic of China ${ }^{2}$ Shanghai Mental Health Center, Shanghai Jiaotong University School of Medicine, 600 South Wanping Road, Shanghai 200030, People's Republic of China

Full list of author information is available at the end of the article
}

supported the presence of co-morbidity of PD traits in subjects with SZ [7-9]. The presence of SZ traits may potentially have enormous impacts on a PD assessment. Despite the prevalence of co-morbidities in SZ, there is a relative dearth of information in the current literature of Chinese population. Moreover, estimates of the prevalence of PD are quite varied across epidemiological surveys in different countries. This ranges between $4.5 \%$ and $100 \%$ [10-12] among patients with SZ and other psychotic disorders.

Furthermore, former investigations present with limitations that may affect a comprehensive understanding of the rates of PD in patients with SZ. The epidemiological survey in this population in the past 2 decades showed no 
persuasive evidence of the co-morbidity between $\mathrm{SZ}$ and PD. This is in part due to inadequate sample sizes and non-representation of target sample. The demographic and clinical profiles could be a critical component in deciding whether there is difference on PD prevalence rates. Several previous studies contained mixed samples (e.g. including schizoaffective disorder and other forms of psychoses). Sample sizes were also generally small, and lacked the inclusion of various clusters and subtypes of PD. Hence it calls for further investigation to address the question of prevalence of PD in SZ.

Both PD and SZ are chronic and debilitating mental disorders. Hence, it is important to collect epidemiological data on the co-morbidity of PD and SZ [13-16]. This data will have practical implications for practicing clinicians $[15,17]$. A recent study indicated that co-morbid Borderline PD has a significant negative longitudinal impact on the course and outcome of patients with SZ [18]. Examining PD in SZ patients will also help us understand the "schizophrenic spectrum" concept, which includes group disorders like SZ and Cluster A PDs (i.e. schizotypal, schizoid, and paranoid PD) that could be genetically inherited [19-21]. As mentioned in previous studies, some PDs traits may be an independent risk syndrome for psychosis [22, 23]. Hence, it is important to identify a possible range of pre-morbid PDs that may occur as part of the prodromal phase of SZ that addresses both symptoms and risks for future psychosis.

It has been recognised that pathological personality occurs in the context of SZ both before and after the onset of psychosis. Hence, several papers put forward the notion that some pre-morbid subtypes of PD (such as schizotypal PD) may be more vulnerable to SZ, and as a result, affect the prognosis of SZ. There is an abundance of evidence that supports the high co-morbidity rate between PD and affective or anxiety disorders [24-27]. Yet, compared to other mental disorders, literature on the co-morbidity of $\mathrm{PD}$ with $\mathrm{SZ}$ is significantly lesser. It appears that clinicians in China also tend to pay less attention to a co-morbid PD diagnosis with patients with SZ.

There are no existing reports on the prevalence of PD in Chinese patients with SZ. Even in the Chinese literature, related data of pathological personality in SZ are rare. Because diagnosis of PD in China adheres to the CCMD-3 standards (Chinese Classification and Diagnostic Criteria of Mental Disorders, Third Edition), other mental disorders recognised by the DSM-IV (Diagnostic and Statistical Manual of Mental Disorders, 4th Edition) are excluded. As a result, the co-morbidity of PDs in SZ in China remains largely unknown. The results of PD rates vary in different countries. For example, the Canadians and Swedish were less likely to meet a diagnosis of PD in the psychotic population compared to the Spanish patients $[11,28]$. The diagnosis of PD may be influenced by cultural and social backgrounds. The Chinese grew up under the strong influence of traditional eastern values while facing rapid changes in the social structure. This has, no doubt, a broad range of impacts on the Chinese community, and hence is worth exploring this phenomenon.

The primary objective of the present study is to examine the distribution of PDs in Chinese patients with SZ. To the best of our knowledge, this is the first study that examines the prevalence of DSM-IV PD using a large clinical sample with SZ in China. It is also an examination of the extent of PD-SZ co-morbidity using DSM diagnoses compared to those with affective disorder or neurosis (ADN). A few hypotheses (such as the pre-morbid model for spectrum hypothesis and the post-morbid model for scar hypothesis) were tested in efforts to enhance our understanding of their inter-relations and differences. In addition, the current study is the first to discuss the feasibility and necessity in carrying out possible comprehensive interventions on pathological personality in SZ population.

\section{Method \\ Subjects}

The epidemiologic survey on PD in clinical settings was conducted in 2006 in Shanghai [29-33]. As detailed elsewhere [30], the participants were recruited from the largest psycho-counseling and psychiatric clinics in Shanghai. Two staff members issued invitation letter to the one-tenth outpatient in psycho-counseling and one-twentieth outpatient in psychiatric clinics according to hospital registration list. Thus, in total of 3402 subjects were randomly sampled from the mental health service setting. During the preliminarily screening process, 327 ineligible outpatients were excluded according to the inclusion and exclusion criteria. After obtaining the patients' informed consent, 3075 subjects were included in the study, and screened with a self-reported questionnaire (the Personality Diagnostic Questionnaire, PDQ-4+). Subsequently, 2590 participants who screened positive were referred for a face-to-face interview (Structured Clinical Interview for DSM-IV AxisII, SCID-II). The CCMD-3 diagnoses were collected according to outpatients' medical records.

For this paper, we obtained data on a selected sample of outpatients with SZ. About 951 outpatients (30.9 \%) were diagnosed with SZ or other psychotic disorders according to their medical records. To ensure that selection is only limited in SZ, clinicians were asked to check the patients' medical records. 101 subjects $(10.6 \%)$ were then excluded because their diagnosis was unclear or other psychotic disorders (such as paranoid psychosis, acute transient psychosis, traveling psychosis et al.).

Consequently, the remaining 850 subjects with SZ who were recruited from the previous epidemiological survey between May and October 2006 were analyzed. Amongst them were 184 subjects $(21.6 \%)$ from the psycho- 
counseling clinic and 666 subjects $(78.4 \%)$ from the psychiatric clinic. There were 371 males (43.6 \%) and 479 females $(56.4 \%)$. The average age was 31.7 years $(\mathrm{SD}=9.8)$. The average course was 85.2 months $(\mathrm{SD}=95.8)$ within the range of 1-480 month. 543 participants $(63.9 \%)$ were single, $238(28.0 \%)$ were married, 54 (6.4) were divorced, 15 (1.8 \%) were widowhood; 323 (38.0 \%) completed college or higher; while 453 (53.3\%) participants earned less than 1000 Yuan a month.

From these 3075 subjects, 1403 outpatients who were diagnosed with affective disorder or neurosis (ADN) were selected as the control group. They were identified through their medical records. Amongst those outpatients, there were 605 males and 798 females, with a mean age of 32.6 years $(\mathrm{SD}=10.2$, ranged $18-60$ years). 742 subjects with mood disorders (including bipolar disorder and depression), 661 subjects with neurosis (including anxiety disorders $(N=517)$ such as phobia, panic disorder, generalized anxiety disorder, obsessive-compulsive disorder, and somatoform disorders $(N=144)$ such as somatization disorder, hypochondriasis, neurasthenia).

\section{Assessment tools of personality disorders and procedures} The Research Ethics Committee at the Shanghai Mental Health Centre approved the study in 2006. All participants were given detailed explanation about the study, including a plain language statement written in their native language. Their written informed consent was obtained before recruited for the study. Only participants of the clinic who were judged to be fully competent to give informed consent for participation were included. An independent senior psychiatric nurse judged if the individual with SZ was competent in giving informed consent. Participation could be withdrawn at any time, and non-participation in the research would not affect the quality of clinical care.

PD assessments were administered using the Personality Diagnostic Questionnaire (PDQ-4+) and Structured Clinical Interview for DSM-IV AxisII(SCID-II)[30]. Both correspond to the criteria of DSM-IV PD. The SCID-II is a semi-structured clinical interview for PD diagnosis. It contains 12 subscales corresponding to the 12 Axis II DSM-IV PDs, which comprise Cluster A PD (Paranoid, Schizoid, Schizotypal PD), Cluster B PD (Histrionic, Narcissistic, Borderline, Antisocial PD), Cluster C PD (Avoidant, Dependent, Obsessive-compulsive PD), Passive-aggressive PD and Depressive PD (in the appendix of DSM-IV). The sample was selected using a two-stage probability sample design. At the first stage, all the eligible subjects was screened by PDQ-4+, which conducted by three senior nurses. At the second stage, patients whose PDQ-4+ score met some form of PD subsequently attended the SCID-II interview by two trained psychiatrists. The Chinese version of SCID-II was previously translated and adapted by the research team members [34].

PDQ-4+ screening questions were administered to 850 subjects with SZ. PDQ-4+ has been widely used to screen for DSM-IV PD. Although specificity is medium, it is a relatively sensitive (0.89) test. 523 subjects whose PDQ-4+ test result was positive were interviewed with the SCID-II. The diagnosis of PDs requires evaluation of long-term patterns of functioning. Hence that implies that there is a potential for state effects. Subjects were asked to reflect and discuss about "everyday life" rather than "recent situations" during PD assessments.

\section{Diagnosis of Schizophrenia and other Axis I disorder}

CCMD-3 (the Chinese Classification of Mental Disorders) is substantially influenced by ICD-10 and DSM-IV representations. The uniaxial classification system used in CCMD gave little thought to issues of co-occurring PDs with other disorders. Most of the diagnostic criteria of SZ are identical to international classifications. However, there is a major difference between the CCMD-3 and DSM systems in the diagnosis of SZ; the duration criterion for SZ is 1 month in CCMD-3, but 6 months in DSM-IV.

Subjects with ADN were considered as a control group because overwhelming clinical evidence showed that depression and neurosis frequently coexist in the same individual, either concurrently or at different times. Therefore, it is hard to distinguish mood and neurosis in detail. This group is also highly heterogeneous with SZ group. Differences include clinical characteristics, presence of predisposing personality, social factors, and the preservation of insight. Amongst the patients in psychiatric clinics and psycho-counseling clinics, SZ, affective disorders and neurosis made up the majority (making up nearly 3/4 of the total sample in 2006).

\section{Statistical analysis}

All analyses presented were conducted for the sample with $\mathrm{SZ}$ and have used ADN as the comparative group. Frequencies and $95 \%$ confidence interval $(95 \% \mathrm{CI})$ of PDs, in accordance to PDQ-4+ and SCID-II were calculated by cluster and specific PD. These comparisons assessed for unadjusted differences by age, sex, education and marriage state, proportions of patients visiting psycho-counseling or psychiatric clinic, self-reported characteristics and course. Odds ratios (OR) were generated to assess associations of PD with those sociodemographic characteristics. Before applying parametric statistics, all variables were checked the normality of the scores distribution.

Two tailed t-tests were used to compare PDQ-4+ mean scores of patients with SZ and ADN by cluster and specific PD. Chi-squared tests were used to compare 
Table 1 Frequency of DSM-IV PDs among patients with schizophrenia using PDQ-4+ and SCID-II

\begin{tabular}{|c|c|c|c|c|}
\hline & \multicolumn{2}{|l|}{ PDQ-4+ } & \multicolumn{2}{|l|}{ SCID-II } \\
\hline & $N(\%)$ & $95 \% \mathrm{Cl}$ & $N(\%)$ & $95 \% \mathrm{Cl}$ \\
\hline${ }^{\mathrm{a} A n y}$ PDs & $693(81.53 \%)$ & $78.92-84.14 \%$ & $204(24.00 \%)$ & $21.13-26.87 \%$ \\
\hline Any Cluster A PD & 503 (59.18 \%) & $55.87-62.48 \%$ & 103 (12.12 \%) & $9.92-14.31 \%$ \\
\hline Paranoid PD (PAR) & 334 (39.29 \%) & $36.01-42.58 \%$ & 65 (7.65 \%) & $5.86-9.43 \%$ \\
\hline Schizoid PD (SCH) & 167 (19.65 \%) & $16.98-22.32 \%$ & $20(2.35 \%)$ & $1.33-3.37 \%$ \\
\hline Schizotypal PD (SCHT) & 314 (36.94 \%) & $33.70-40.19 \%$ & 37 (4.35\%) & $2.98-5.72 \%$ \\
\hline Any Cluster B PD & 424 (49.88 \%) & $46.52-53.24 \%$ & 32 (3.76 \%) & $2.49-5.04 \%$ \\
\hline Histrionic PD (HIS) & 254 (29.88 \%) & $26.81-32.96 \%$ & 10 (1.18 \%) & $0.45-1.90 \%$ \\
\hline Narcissistic PD (NAR) & 220 (25.88 \%) & $22.94-28.83 \%$ & $9(1.06 \%)$ & $0.37-1.75 \%$ \\
\hline Borderline PD (BOR) & 322 (37.88 \%) & $34.62-41.14 \%$ & 17 (2.00 \%) & $1.06-2.94 \%$ \\
\hline Antisocial PD (ANT) & 215 (25.29 \%) & $22.37-28.22 \%$ & $2(0.24 \%)$ & $0-0.56 \%$ \\
\hline Any Cluster C PD & $580(68.24 \%)$ & $65.11-71.37 \%$ & $102(12.00 \%)$ & $9.82-14.18 \%$ \\
\hline Avoidant PD (AVO) & 447 (52.59 \%) & $49.23-55.95 \%$ & 64 (7.53 \%) & $5.76-9.30 \%$ \\
\hline Dependent PD (DEP) & 254 (29.88 \%) & $26.81-32.96 \%$ & 29 (3.41\%) & $2.19-4.63 \%$ \\
\hline Obsessive-compulsive PD (OBC) & 410 (48.24 \%) & $44.88-51.59 \%$ & 30 (3.53\%) & $2.29-4.77 \%$ \\
\hline \multicolumn{5}{|l|}{ In the Appendix of DSM-IV } \\
\hline Passive-aggressive PD (PAG) & $292(34.35 \%)$ & $31.16-37.55 \%$ & $23(2.71 \%)$ & $1.62-3.80 \%$ \\
\hline Depressive PD (DPS) & 259 (30.47\%) & $27.38-33.56 \%$ & $44(5.18 \%)$ & $3.69-6.67 \%$ \\
\hline
\end{tabular}

${ }^{\mathrm{a}}$ Only includes 12 PDs as stated in the DSM-IV, not include PD not otherwise specified (PD NOS)

the proportions of PD patients according to SCID-II. All statistical differences were considered significant at $P<$ 0.05. Stepwise regression modeling was used to assess the impact of PD on a mental disorder diagnosis. The presence of SZ was entered as the dependant variable and age, sex and the presence of PD were entered as the independent variables. OR, $95 \% \mathrm{CI}, \chi^{2}$ statistic and $p$ value for all individual variables in the final model were estimated.

\section{Results}

\section{Prevalence of PDs}

When the self-rating tool of PDQ-4+ was used for described the feature of PDs, the rate of endorsement of PD traits in our schizophrenics sample with was relatively high (Table 1). $81.5 \%$ participants presented with at least one PD traits. The most frequent PD trait in this sample was avoidant PD (52.6\%), followed by obsessive-compulsive (48.2\%) and paranoid PD (39.3 \%).

When structured interview tool of SCID-II were used for PD diagnosis, the frequency of PD among respondents with SZ was also common. Nearly one quarter of participants meet criteria for at least one DSM-IV PD, with the most prevalent PD was paranoid PD (7.65\%), followed by avoidant (7.53 \%) and schizotypal (4.35\%) PD. Cluster A PDs (12.12\%) were the most common PD types compared to other clusters.

\section{Association with clinical and socio-demographic characteristics}

The overall prevalence of PDs was significantly greater in younger group than older $(\mathrm{OR}=1.969)$ (Table 2). Subjects who were single $(\mathrm{OR}=1.812)$, had a greater prevalence compared to married status. PDs were also significantly more prevalent in the psycho-counseling clinic $(\mathrm{OR}=$ 2.438) than in Psychiatric clinic. The prevalence of PD also varied across categories of self-reported characteristics (Introversion, Middle type, Extroversion). PDs were most prevalent in subjects who had characteristics of Introversion $(\mathrm{OR}=1.982)$. Subjects who had an ill duration of lesser than 6 months were more prevalent compared to those with a more chronic presentation, but the odds of having PD did not differ significantly.

\section{Comparative analyses of personality disturbance}

As seen in Table 3, the first set of analyses focused on the self-reported personality disturbance. Subjects with SZ reported significantly less symptoms of cluster B and $\mathrm{C}$ PD traits compared to subjects with ADN, except the dependent PD. The mean score of cluster A PDs of two groups did not differ significantly. The second set of analyses was conducted to investigate the difference of PD prevalence between the SZ and ADN group. Subjects with SZ were significantly more likely to have schizotypal PD (4.4 \% vs. $2.1 \%, p=0.003)$ and paranoid PD (7.6 \% vs. $5.4 \%, p=0.034)$, but much less likely to have 
Table 2 Odds ratios (and $95 \% \mathrm{Cl}$ ) of having PDs, by clinical and socio-demographic characteristics

\begin{tabular}{|c|c|c|c|c|c|c|}
\hline & Number & PD & PD (\%) & $95 \% \mathrm{Cl}$ & OR & $95 \% \mathrm{Cl}$ \\
\hline Male & 371 & 86 & 23.2 & $18.9 \sim 27.5 \%$ & 0.923 & $0.671-1.269$ \\
\hline Female & 479 & 118 & 24.6 & $20.8 \sim 28.5 \%$ & 1.083 & $0.788-1.489$ \\
\hline 20 Years & 439 & 131 & 29.8 & $25.6 \sim 34.1 \%$ & 1.969 & $1.423-2.726$ \\
\hline $30 \sim$ Years & 411 & 73 & 17.8 & $14.1 \sim 21.5 \%$ & 0.508 & $0.367-0.703$ \\
\hline Middle or high school & 527 & 127 & 24.1 & $20.4 \sim 27.8 \%$ & 1.014 & $0.733-1.403$ \\
\hline College or higher & 323 & 77 & 23.8 & $19.2 \sim 28.5 \%$ & 0.986 & $0.713-1.364$ \\
\hline Single or divorced, widowhood & 612 & 164 & 26.8 & $23.3 \sim 30.3 \%$ & 1.812 & $1.234-2.660$ \\
\hline Married & 238 & 40 & 16.8 & $12.1 \sim 21.6 \%$ & 0.552 & $0.376-0.810$ \\
\hline Psycho-counseling clinic & 184 & 70 & 38.0 & $31.0 \sim 45.1 \%$ & 2.438 & $1.713-3.469$ \\
\hline Psychiatric clinic & 666 & 134 & 20.1 & $17.1 \sim 23.2 \%$ & 0.410 & $0.288-0.584$ \\
\hline Introversion & 438 & 131 & 29.9 & $25.6 \sim 34.2 \%$ & 1.982 & $1.431-2.743$ \\
\hline Middle type & 293 & 53 & 18.1 & $13.7 \sim 22.5 \%$ & 0.594 & $0.418-0.844$ \\
\hline Extroversion & 119 & 20 & 16.8 & $10.1 \sim 23.5 \%$ & 0.601 & $0.361-0.999$ \\
\hline Course $<=6$ month & 151 & 45 & 29.8 & $22.5 \sim 37.1 \%$ & 1.442 & $0.975-2.132$ \\
\hline Course $>6$ month & 699 & 159 & 22.7 & $19.6 \sim 25.9 \%$ & 0.694 & $0.469-1.025$ \\
\hline
\end{tabular}

Note: Age grouped by median age of the sample

borderline, obsessive-compulsive, depressive, narcissistic and histrionic PD.

\section{Stepwise regression analyses}

Stepwise regression was employed in an attempt to identify the risk factors of PDs related to SZ or ADN. Logistic regression (forward stepwise) analyses were performed in the presence of SZ as the dependent variable while age, gender, different type of PDs acted as independent variables (Table 4). Among those PDs, paranoid and schizotypal PD were significant predictors of SZ, and borderline, obsessive-compulsive, depressive, histrionic PDs were significant predictors of ADN.

Table 3 Comparison of difference between SZ and ADN for PDQ-4+ PDs scores and SCID-II PDs frequency

\begin{tabular}{|c|c|c|c|c|c|c|}
\hline & \multicolumn{3}{|c|}{ PDQ-4+ } & \multicolumn{3}{|l|}{ SCID-II } \\
\hline & $\overline{\mathrm{SCH}}$ & ADN & $t$ value & $\overline{\mathrm{SCH}}(\%)$ & ADN (\%) & $x^{2}$ value \\
\hline Any Cluster A PD & 9.04 & 9.04 & $-0.028^{\mathrm{a}}$ & $12.1 \%$ & $9.6 \%$ & 0.054 \\
\hline PAR & 2.82 & 2.97 & -1.759 & $7.6 \%$ & $5.4 \%$ & $0.034^{*}$ \\
\hline $\mathrm{SCH}$ & 2.39 & 2.41 & $-.308^{a}$ & $2.4 \%$ & $2.6 \%$ & 0.677 \\
\hline $\mathrm{SCHT}$ & 3.82 & 3.66 & 1.648 & $4.4 \%$ & $2.1 \%$ & $0.003^{* *}$ \\
\hline Any Cluster B PD & 12.32 & 13.05 & $-2.498^{\mathrm{a} *}$ & $3.8 \%$ & $12.2 \%$ & $0.000^{* *}$ \\
\hline HIS & 3.34 & 3.64 & $-3.705^{* *}$ & $1.2 \%$ & $3.0 \%$ & $0.005^{* *}$ \\
\hline NAR & 3.10 & 3.38 & $-3.084^{* *}$ & $1.1 \%$ & $2.9 \%$ & $0.004^{* *}$ \\
\hline BOR & 4.06 & 4.44 & $-3.472^{* *}$ & $2.0 \%$ & $7.6 \%$ & $0.000^{* *}$ \\
\hline ANT & 1.82 & 1.60 & $3.155^{* *}$ & $0.2 \%$ & $0.4 \%$ & 0.457 \\
\hline Any Cluster C PD & 10.60 & 11.26 & $-3.105^{* *}$ & $12.0 \%$ & $21.7 \%$ & $0.000^{* *}$ \\
\hline AVO & 3.61 & 3.89 & $-3.298^{* *}$ & $7.5 \%$ & $9.8 \%$ & 0.063 \\
\hline DEP & 3.32 & 3.41 & $-.998^{a}$ & $3.4 \%$ & $3.1 \%$ & 0.720 \\
\hline $\mathrm{OBC}$ & 3.66 & 3.95 & $-3.467^{\mathrm{a} * *}$ & $3.5 \%$ & $10.8 \%$ & $0.000^{* *}$ \\
\hline \multicolumn{7}{|c|}{ In the Appendix of DSM-IV } \\
\hline PAG & 2.77 & 2.97 & $-2.740^{* *}$ & $2.7 \%$ & $4.2 \%$ & 0.065 \\
\hline DPS & 3.35 & 3.89 & $-5.987^{* *}$ & $5.2 \%$ & $11.4 \%$ & $0.000^{* *}$ \\
\hline
\end{tabular}

aLevene's Test for Equality of Variances is significant; ${ }^{*} p<0.05 ;{ }^{* *} p<0.01$ 
Table 4 Forward stepwise logistic regression for risk factors predicting the clinical diagnoses

\begin{tabular}{lclllrc}
\hline Variable & Beta & S.E. & OR & $95 \% \mathrm{Cl}$ & $x^{2}$ statistic & $P$ value \\
\hline Age & 0.011 & 0.005 & 1.011 & $1.003-1.020$ & 6.335 & 0.012 \\
PAR & -0.958 & 0.205 & 0.384 & $0.257-0.573$ & 21.841 & 0.000 \\
SCHT & -0.705 & 0.271 & 0.494 & $0.291-0.839$ & 6.800 & 0.009 \\
BOR & 1.457 & 0.284 & 4.293 & $2.461-7.491$ & 26.320 & 0.000 \\
HIS & 0.864 & 0.362 & 2.373 & $1.168-4.821$ & 5.704 & 0.017 \\
OBC & 1.307 & 0.214 & 3.695 & $2.427-5.625$ & 37.134 & 0.000 \\
DPS & 0.761 & 0.186 & 2.140 & $1.486-3.082$ & 16.715 & 0.000 \\
Constant & -0.006 & 0.155 & 0.994 & - & 0.002 & 0.967 \\
\hline
\end{tabular}

\section{Discussion}

SZ has always been considered the most serious mental illness in China, and usually accompanied with stigma or fear from the general community. The personality of this population is indeed difficult to understand and hence, is often neglected by people around them. Even psychiatric professionals only identify with symptoms such as hallucinations and delusions, and little attention has been given to the co-occurrence of PDs in the clinical assessment routine. This study is a groundbreaking survey of PDs among patients with SZ in the Chinese population. More than $80 \%$ of subjects presented with at least one PD trait, and about one quarter of outpatients suffer from at least one diagnosable PD. Substantial evidence suggests that PDs influence other mental disorders' prognosis, hence to a certain degree, overlook the PD in patients with SZ might missed the chance of predicting an Axis I prognosis and treatment response. Consequently, opportunities of a focused approach to treatment using psychotherapy are lost.

The prevalence of PD was $24.0 \%$ in this Chinese clinical population with $\mathrm{SZ}$, and was within the range of estimates (22- $28 \%$ ) found in some previous epidemiologic surveys [35-37], but not in others [7, 38-40]. Newton-Howes and colleagues (2008) used the multilevel modeling method and found that there was great variation in the reported prevalence of PD in SZ. The multilevel modeling method identified that the most possible reason for this difference was that these studies were carried out in different countries. Other possibilities that may also have contributed to the discrepancies included sample size, differences in diagnostic criteria, assessment instruments, survey designs and methodologies.

After the SCID-II interview, Cluster A PDs (especially Paranoid PD) became the most prevalent PD compared to other PDs. That is, this pattern of PD co-morbidity may be explained by the spectrum hypothesis. Kraepelin and Bleuler considered schizophrenia as the worsening of schizoid features in the early 20th century. In the late 20th-century, Cluster A PDs was considered as SZ spectrum PDs [41-43]. It is also well known that Cluster
A PDs was more likely to be noticed among relatives of patients with SZ $[44,45]$. In addition, Cluster C PDs were also very common among those subjects. The finding that self-reported Cluster C PDs pathology (especially Avoidant and Obsessive-compulsive PD) is more common in Chinese patients with SZ is also consistent with the results of previous studies $[28,46]$. This may be partly due to the effects of SZ on personality over time (the scar hypothesis). The experiences of stigma and social disability might account for 'fearful' group traits. This paper also analyzed the socio-demographic characteristics of patients with SZ. Taken as a whole, the factors associated with increased odds of PD were younger age, non-marriage status (single, divorced, or widowhood), in psycho-counseling clinic, and self-reported characteristics of introversion. This was consistent with findings from many prior studies, which identified that PDs were more prevalent among younger adults than older adults [47-50]. Although PDs are defined as stable and enduring patterns in the DSM, many previous PD surveys proposed that PD traits (especially Borderline PD traits) remit with age $[51,52]$. Thus, from another viewpoint, this phenomenon is partly supported the scar hypothesis, which emphasized the influence of the presence of SZ on the features in personality psychopathology across the lifespan. Other characteristics might reflect that those schizophrenic patients with PDs live a solitary life, required more support and attention from the society, family and mental health professionals.

When compared to the patients with $\mathrm{ADN}$, we found a distinct high co-morbidity of 'eccentric' group PDs amongst patients with SZ, while 'emotional' and 'fearful' group PDs were less commonly co-morbid. Paranoid and schizotypal PD seemed to be strongly associated with SZ, but borderline, obsessive-compulsive, depressive, histrionic PD were predictive of $\mathrm{ADN}$. The results of this study showed that PD plays an important role in accurately diagnosing Axis I disorder. Because this was not a longitudinal study, it was difficult to determine the relation of consequences to antecedent. Hence, we mainly considered several possibilities with regard to the different pathological features of PDs among Axis I disorders: 1) certain cluster of pre-morbid PD may be earlier expressions of symptomatology in Axis I disorders; 2) certain cluster of pre-morbid PD may be vulnerabilities to SZ or ADN; 3) certain cluster of co-morbid PD may be the effects of the presence of Axis I disorders on personality over time.

The pattern of co-morbidity and heterogeneity of PD in $\mathrm{SZ}$, as reported in this paper, may have several impacts on clinical practice. For example, it highlights the need for Chinese psychiatrists to pay attention to the following points. Firstly, as approximately one quarter of the recruited sample had been diagnosed with PD, detailed PD assessments and interventions should be considered for patients with SZ. That information will also be used to 
help us to improve the effectiveness and efficacy of the care provided at mental health service. Secondly, high comorbid rates of Cluster A PDs in SZ imply that subjects who fall in the schizophrenic spectrum PD may be strongly linked to SZ. Therefore, clinicians need to give attention to Cluster A PD-oriented therapy in order to minimize the risk of psychosis and long term sequelae. Lastly, the difference of comorbid PD between SZ and ADN suggests that there may be different ways of developing therapeutic alliance during therapy. Help-seeking behavior and medical resource utilization may be quite different with personality types. Therefore, a range of mental health services and social support may help meet the different needs for prevention and rehabilitation.

Several methodological limitations must be considered in regards to this study. The principal limitation of the crosssectional design lies in its inability to distinguish between cause and effect of PD and SZ. The diagnosis of SZ in the study was made according to CCMD-3, which may then result in a fundamental difference in results of this sample with other international studies. PD symptoms, somehow, are a part of the syndrome of SZ, which may affect the accuracy of personality assessment. Subjects in this study were outpatients of the largest mental health service in Shanghai and therefore, this may introduce selection bias and reduce the generalizability of our findings to the general SZ population (i.e., recruitment from a general psychiatric hospital or forensic psychiatric hospital).

\section{Conclusions}

Despite these limitations, these data provide an important step in understanding the rates of PDs among Chinese patients with SZ, as well as the association between PD and SZ. Furthermore, it helps in understanding the role of PD plays in the difference between SZ and ADN. Future research can consider following up with patients diagnosed with PD to ascertain how they are capable of acting on or influencing each other.

\section{Abbreviations \\ ADN, affective disorder or neurosis; ANT, antisocial PD; AVO, avoidant PD; BOR, borderline PD; CCMD-3, chinese classification and diagnostic criteria of mental disorders, Third Edition; DEP, dependent PD; DPS, depressive PD; DSM-IV, diagnostic and statistical manual of mental disorders, 4th Edition; HIS, histrionic PD; NAR, narcissistic PD; OBC, obsessive-compulsive PD; OR, odds ratio; PAG, passive-aggressive PD; PAR, paranoid PD; PD, personality disorder; PDQ-4+, personality diagnostic questionnaire, 4+ Edition; SCH, schizoid PD; SCHT, schizotypal PD; SCID-II, structured clinical interview for DSM-IV Axisll; SZ, schizophrenia.}

\section{Acknowledgements}

The authors wish to thank the participants and study team in Shanghai Mental Health Centre (SMHC).

\section{Funding}

This study was supported by National Natural Science Foundation of China (81201043, 81372122, 81261120410, 81361120403) (the design of the study and collection), Shanghai Science and Technology Committee (15411967200,
14411961400) (the design of the study and collection), The 12th Five-Year projects of PLA research (NO.13QJ003-005) (analysis and interpretation of data), Mental Health Application Research in PLA(NO.12XLZ109) (analysis and interpretation of data), National Key Clinical Disciplines at Shanghai Mental Health Center (OMA-MH, 2011-873) (analysis and interpretation of data), Shanghai education science research project(No.B13028) (analysis and interpretation of data), Shanghai Key Laboratory of Psychotic Disorders (13dz2260500) (analysis and interpretation of data), Shanghai Jiao Tong University Foundation (14JCRY04, YG2014MS40) (write and revise the manuscript). It was also supported by SHSMU-ION Research Center for Brain Disorders (2015NKX001) (write and revise the manuscript), Program of Shanghai Academic Research Leader(16XD1402400) (write and revise the manuscript) and Shanghai health system advanced appropriate technology (2013SY003) (write and revise the manuscript).

\section{Availability of data and materials}

Due to the conflict with patients' privacy (it was not in accordance with patients' written informed consent), data will not be shared.

\section{Authors' contribution}

THZ conceived the idea of this study, wrote the first draft of manuscript, conducted the statistical analyses and involved in clinical and diagnostic assessments. YYW and YXT hepled in writing the first draft of manuscript, completely revised the manuscript and re-analyzed the data. $A C, L H X$, and $Y Y T$ helped in the design of the study and edited the manuscript. THZ, XHL and YFD interviewed participants and collected and organized the primary data. $\mathrm{KDJ}, \mathrm{ZPX}$, and JJW the study and provided supervision in the implementation of the study. TS, XP, YC, and ZQL contributed in the revision of the manuscript, helped in re-analyzed the data. All authors have approved the final manuscript.

\section{Competing interests}

The authors declare that they have no competing interests.

\section{Consent for publication}

Not applicable.

\section{Ethics approval and consent to participate}

The Research Ethics Committee at the Shanghai Mental Health Centre (SMHC) approved the study. The written informed consent was obtained from all participants before being recruited for the study. All experiments were in compliance with the Helsinki declaration.

\section{Author details}

'Department of Medical Psychology, Faculty of Mental Health, Second Military Medical University, Shanghai 200433, People's Republic of China. ${ }^{2}$ Shanghai Mental Health Center, Shanghai Jiaotong University School of Medicine, 600 South Wanping Road, Shanghai 200030, People's Republic of China. ${ }^{3}$ Department of Psychological Medicine, Changi General Hospital, Singapore, Singapore. ${ }^{4}$ Shanghai Key Laboratory of Psychotic Disorders (No.13dz2260500), Bio-X Institutes, Key Laboratory for the Genetics of Developmental and Neuropsychiatric Disorders (Ministry of Education), Shanghai, People's Republic of China.

Received: 5 July 2015 Accepted: 22 June 2016

Published online: 08 July 2016

\section{References}

1. Cadenhead KS, Braff DL. Endophenotyping schizotypy: a prelude to genetic studies within the schizophrenia spectrum. Schizophr Res. 2002;54(1-2):47-57.

2. Hazlett $E A$, Goldstein KE, Kolaitis JC. A review of structural MRI and diffusion tensor imaging in schizotypal personality disorder. Curr Psychiatry Rep. 2012;14(1):70-8.

3. Moore EA, Green MJ, Carr VJ. Comorbid personality traits in schizophrenia: Prevalence and clinical characteristics. J Psychiatr Res. 2012;46(3):353-9.

4. Rossi A, Daneluzzo E. Schizotypal dimensions in normals and schizophrenic patients: a comparison with other clinical samples. Schizophr Res. 2002; 54(1-2):67-75

5. Schultze-Lutter F, Klosterkotter J, Michel C, Winkler K, Ruhrmann S. Personality disorders and accentuations in at-risk persons with and without conversion to first-episode psychosis. Early Interv Psychiatry. 2012;64(4):389-98. 
6. Fanous AH, Neale MC, Gardner CO, Webb BT, Straub RE, O'Neill FA, Walsh D, Riley BP, Kendler KS. Significant correlation in linkage signals from genome-wide scans of schizophrenia and schizotypy. Mol Psychiatry. 2007;12(10):958-65.

7. McMillan KA, Enns MW, Cox BJ, Sareen J. Comorbidity of Axis I and II mental disorders with schizophrenia and psychotic disorders: findings from the National Epidemiologic Survey on Alcohol and Related Conditions. Can J Psychiatry. 2009;54(7):477-86

8. Pulay AJ, Stinson FS, Dawson DA, Goldstein RB, Chou SP, Huang B, Saha TD, Smith SM, Pickering RP, Ruan WJ. Prevalence, correlates, disability, and comorbidity of DSM-IV schizotypal personality disorder: results from the wave 2 national epidemiologic survey on alcohol and related conditions. Prim Care Companion J Clin Psychiatry. 2009;11(2):53-67.

9. Camisa KM, Bockbrader MA, Lysaker P, Rae LL, Brenner CA, O'Donnell BF. Personality traits in schizophrenia and related personality disorders. Psychiatry Res. 2005;133(1):23-33.

10. Caton CL, Shrout PE, Eagle PF, Opler LA, Felix A, Dominguez B. Risk factors for homelessness among schizophrenic men: a case-control study. Am J Public Health. 1994;84(2):265-70.

11. Newton-Howes $G$, Tyrer $P$, North B, Yang M. The prevalence of personality disorder in schizophrenia and psychotic disorders: systematic review of rates and explanatory modelling. Psychol Med. 2008;38(8):1075-82.

12. Oldham JM, Skodol AE, Kellman HD, Hyler SE, Doidge N, Rosnick L, Gallaher PE. Comorbidity of axis I and axis II disorders. Am J Psychiatry. 1995;152(4):571-8.

13. Tyrer P, Manley C, Van Horn E, Leddy D, Ukoumunne OC. Personality abnormality in severe mental illness and its influence on outcome of intensive and standard case management: a randomised controlled trial. Eur Psychiatry. 2000;15 Suppl 1:7-10.

14. Tyrer $P$, Simmonds S. Treatment models for those with severe mental illness and comorbid personality disorder. Br J Psychiatry Suppl. 2003:44:S15-18.

15. Smith TE, Grabstein $E$, Kentros M, Kulchycky S, Borgaro S. Axis II diagnoses and treatment refractoriness in schizophrenia. Psychiatr Q. 1996;67(1):51-64.

16. Smith TE, Deutsch A, Schwartz F, Terkelsen KG. The role of personality in the treatment of schizophrenic and schizoaffective disorder inpatients: a pilot study. Bull Menninger Clin. 1993;57(1):88-99.

17. Gandhi N, Tyrer P, Evans K, McGee A, Lamont A, Harrison-Read P. A randomized controlled trial of community-oriented and hospital-oriented care for discharged psychiatric patients: influence of personality disorder on police contacts. J Pers Disord. 2001;15(1):94-102.

18. Bahorik AL, Eack SM. Examining the course and outcome of individuals diagnosed with schizophrenia and comorbid borderline personality disorder. Schizophr Res. 2010;124(1-3):29-35.

19. Raballo A, Parnas J. The silent side of the spectrum: schizotypy and the schizotaxic self. Schizophr Bull. 2011;37(5):1017-26.

20. Krause V, Krastoshevsky O, Coleman MJ, Bodkin JA, Lerbinger J, Boling L, Johnson F, Gibbs A, Cole JO, Huang Z. Tailoring the definition of the clinical schizophrenia phenotype in linkage studies. Schizophr Res. 2010;116(2-3):133-42.

21. Torgersen S, Edvardsen J, Oien PA, Onstad S, Skre I, Lygren S, Kringlen E. Schizotypal personality disorder inside and outside the schizophrenic spectrum. Schizophr Res. 2002;54(1-2):33-8.

22. Woods SW, Addington J, Cadenhead KS, Cannon TD, Cornblatt BA, Heinssen R, Perkins DO, Seidman LJ, Tsuang MT, Walker EF. Validity of the prodromal risk syndrome for first psychosis: findings from the North American Prodrome Longitudinal Study. Schizophr Bull. 2009;35(5):894-908.

23. Bedwell JS, Donnelly RS. Schizotypal personality disorder or prodromal symptoms of schizophrenia? Schizophr Res. 2005;80(2-3):263-9.

24. Schiavone P, Dorz S, Conforti D, Scarso C, Borgherini G. Comorbidity of DSM-IV Personality Disorders in unipolar and bipolar affective disorders: a comparative study. Psychol Rep. 2004;95(1):121-8.

25. Luca M, Luca A, Calandra C. Borderline Personality Disorder and Depression: An Update. Psychiatr Q. 2011;83(3):281-92.

26. Bienvenu OJ, Stein MB. Personality and anxiety disorders: a review. J Pers Disord. 2003:17(2):139-51.

27. Mancebo MC, Eisen JL, Grant JE, Rasmussen SA. Obsessive compulsive personality disorder and obsessive compulsive disorder: clinical characteristics, diagnostic difficulties, and treatment. Ann Clin Psychiatry. 2005;17(4):197-204.

28. Rodriguez Solano JJ, Gonzalez De Chavez M. Premorbid personality disorders in schizophrenia. Schizophr Res. 2000;44(2):137-44.

29. Zhang $T$, Chow A, Wang L, Dai Y, Xiao Z. Role of childhood traumatic experience in personality disorders in China. Compr Psychiatry. 2012;53(6):829-36.

30. Zhang T, Wang L, Good MJ, Good BJ, Chow A, Dai Y, Yu J, Zhang H, Xiao Z. Prevalence of personality disorders using two diagnostic systems in psychiatric outpatients in Shanghai, China: a comparison of uni-axial and multi-axia formulation. Soc Psychiatry Psychiatr Epidemiol. 2012;47(9):1409-17.

31. Zhang TH, Xiao ZP, Wang LL, Dai YF, Zhang HY, Qiu JY, Tao MY, Wang Z, Wang $\mathrm{X}, \mathrm{Yu} \mathrm{JH}$. A cross-sectional survey on personality disorder in mental disorder outpatients in Shanghai. Zhonghua Liu Xing Bing Xue Za Zhi. 2010;31(8):933-7.

32. Zhang TH, Chow A, Wang LL, Yu JH, Dai YF, Lu X, Good MJ, Good BJ, Xiao ZP. Childhood maltreatment profile in a clinical population in China: A further analysis with existing data of an epidemiologic survey. Compr Psychiatry. 2013;54(7):856-64

33. Zhang T, Good MJ, Good BJ, Chow A, Wang L, Dai Y, Xiao Z. Age and remission of personality pathology in the psychotic disorders compared to mood and/or anxiety disorders. Int J Psychiatry Med. 2012;44(3):241-55.

34. Dai YF, Xiao ZP, Wang Z, Zhang HY, Chen J, Zou Z, He YL, Zhao JC, Zhang MY. The reliability and validity of a Chinese version of the Structured Clinical Interview for DSM-IV personality disorders. Shanghai Archives of Psychiatry. 2006;18(1):1-4.

35. Moran P, Hodgins S. The correlates of comorbid antisocial personality disorder in schizophrenia. Schizophr Bull. 2004;30(4):791-802.

36. Moran P, Walsh E, Tyrer P, Burns T, Creed F, Fahy T. Impact of comorbid personality disorder on violence in psychosis: report from the UK700 trial. Br J Psychiatry. 2003;182:129-34.

37. Caton CL, Shrout PE, Dominguez B, Eagle PF, Opler LA, Cournos F. Risk factors for homelessness among women with schizophrenia. Am J Public Health. 1995;85(8 Pt 1):1153-6.

38. Lindstrom E, Von Knorring L, Ekselius L. Self-reported personality disorders in patients with schizophrenia and the relationship to symptoms, side effects, and social functioning. Nordic J Psychiat. 2000;54(5):341-6.

39. Mueser KT, Rosenberg SD, Drake RE, Miles KM, Wolford G, Vidaver R, Carrieri K. Conduct disorder, antisocial personality disorder and substance use disorders in schizophrenia and major affective disorders. Journal of Studies on Alcohol. 1999:60(2):278-84.

40. Oulis P, Lykouras L, Hatzimanolis J, Tomaras V. Comorbidity of dsm-iii-r personality disorders in schizophrenic and unipolar mood disorders: a comparative study. Eur Psychiatry. 1997;12(6):316-8.

41. Kendler KS, Gruenberg AM, Strauss JS. An independent analysis of the Copenhagen sample of the Danish adoption study of schizophrenia. II. The relationship between schizotypal personality disorder and schizophrenia. Arch Gen Psychiatry. 1981;38(9):982-4.

42. Gunderson JG, Siever LJ. Relatedness of schizotypal to schizophrenic disorders: editors' introduction. Schizophr Bull. 1985;11(4):532-7.

43. Torgersen S. Relationship of schizotypal personality disorder to schizophrenia: genetics. Schizophr Bull. 1985;11(4):554-63.

44. Fogelson DL, Nuechterlein KH, Asarnow RF, Payne DL, Subotnik KL, Giannini CA. The factor structure of schizophrenia spectrum personality disorders: signs and symptoms in relatives of psychotic patients from the UCLA family members study. Psychiatry Res. 1999;87(2-3):137-46.

45. Joober R, Rouleau GA, Lal S, Bloom D, Lalonde P, Labelle A, Benkelfat C. Increased prevalence of schizophrenia spectrum disorders in relatives of neurolepticnonresponsive schizophrenic patients. Schizophr Res. 2005;77(1):35-41.

46. Fogelson DL, Nuechterlein $\mathrm{KH}$, Asarnow RA, Payne DL, Subotnik KL, Jacobson KC, Neale MC, Kendler KS. Avoidant personality disorder is a separable schizophrenia-spectrum personality disorder even when controlling for the presence of paranoid and schizotypal personality disorders The UCLA family study. Schizophr Res. 2007;91(1-3):192-9.

47. Jackson HJ, Burgess PM. Personality disorders in the community: a report from the Australian National Survey of Mental Health and Wellbeing. Soc Psychiatry Psychiatr Epidemiol. 2000;35(12):531-8.

48. Ames A, Molinari V. Prevalence of personality disorders in community-living elderly. J Geriatr Psychiatry Neurol. 1994;7(3):189-94.

49. Bernstein DP, Cohen P, Velez CN, Schwab-Stone M, Siever LJ, Shinsato L. Prevalence and stability of the DSM-III-R personality disorders in a communitybased survey of adolescents. Am J Psychiatry. 1993;150(8):1237-43.

50. Johnson JG, Cohen P, Kasen S, Skodol AE, Hamagami F, Brook JS. Agerelated change in personality disorder trait levels between early adolescence and adulthood: a community-based longitudinal investigation. Acta Psychiatr Scand. 2000;102(4):265-75.

51. Lenzenweger MF, Johnson MD, Willett JB. Individual growth curve analysis illuminates stability and change in personality disorder features: the longitudinal study of personality disorders. Arch Gen Psychiatry. 2004;61(10):1015-24.

52. Torgersen S. The nature (and nurture) of personality disorders. Scand J Psychol. 2009;50(6):624-32. 